PROCEEDINGS OF THE

AMERICAN MATHEMATICAL SOCIETY

Volume 130, Number 11, Pages 3249-3254

S 0002-9939(02)06704-7

Article electronically published on June 11, 2002

\title{
FIXED POINTS FOR SOME NON-OBVIOUSLY CONTRACTIVE OPERATORS
}

\author{
E. DE PASCALE AND L. DE PASCALE
}

(Communicated by Jonathan M. Borwein)

\begin{abstract}
The use of K-normed spaces gives us the possibility to prove that a fixed point theorem due to B. Luo is equivalent to the Banach Contraction Principle. This confirms the conspiracy among fixed point theorems. Moreover the theorem of Lou is improved and extended to different contexts. A counterexample about the fixed points of the sum of a contraction and an integral operator is given. The usefulness of K-norm is tested on a Volterra integral equation as well.
\end{abstract}

\section{INTRODUCTION}

Let $E$ be a real Banach space with norm $\|\cdot\|, I=[0, T](T>0)$. In [1] the author proved the following theorem.

Theorem 1.1. Let $F$ be a closed subset of $C(I, E)$ and $A: F \rightarrow F$ an operator. If there exist $\alpha, \beta \in[0,1), K \geq 0$ such that for any $u, v \in F$,

$$
\|A u(t)-A v(t)\| \leq \beta\|u(t)-v(t)\|+\frac{K}{t^{\alpha}} \int_{0}^{t}\|u(s)-v(s)\| d s, \quad \forall t \in(0, T],
$$

then $A$ has exactly one fixed point $u^{*}$ in $F$. For any $x_{0} \in F$, the iterative sequence $x_{n}=A x_{n-1}$ converges to $u^{*}$ in $F$ and for all $s>0$,

$$
\left\|x_{n}-u^{*}\right\|=o\left(n^{-s}\right) \text { as } n \rightarrow \infty .
$$

The author shows that Theorem 1.1 is a generalization of the Banach Contraction Principle and moreover in Remark 2.2 he claims that the Contraction Principle cannot replace Theorem 1.1 to obtain his results.

Here we want to prove that by using the Contraction Principle much more can be obtained. We do this by using a version of the Contraction Principle in the context of K-normed spaces.

Let $X$ be a linear space over $\mathbb{R}$ or $\mathbb{C}$ and $\left(B,\|\cdot\|_{B}\right)$ a Banach space ordered by means of a cone $\mathbb{K}$. A K-norm is a function $] \cdot[: X \rightarrow \mathbb{K}$ having the usual properties of a norm. $B$ is usually called the norming space. The K-normed space $X$ is said to be complete when it is complete with respect to the norm defined by $\left.\|x\|_{X}:=\|\right] x\left[\|_{B}\right.$.

Received by the editors June 5, 2001

2000 Mathematics Subject Classification. Primary 47H10, 45D05.

Key words and phrases. Fixed points, iterative sequences, K-normed spaces, positive operators, normal cone, contraction operators. 
In the last fifty years the Banach Contraction Principle has been extended to the context of K-normed spaces (and to much more general structures) by various authors. For a survey on the subject we refer to [2] and the bibliography therein.

We recall here the simplest, although stronger, formulation of the above generalizations, since it is enough for our purposes.

Definition 1.2. An operator $A: F \rightarrow F, F$ a closed subset of a K-normed space $X$, is said to be a contraction (or generalized contraction) if there exists a positive linear operator $T: B \rightarrow B$, with spectral radius $\rho(T)<1$, such that for any $u, v \in F$

$$
] A u-A v[\leq T(] u-v[) .
$$

Theorem 1.3. Let $A: F \rightarrow F$ be a generalized contraction of a closed subset $F$ of a K-normed space $X$. Suppose that the cone $\mathbb{K}$ is normal (i.e. $0 \leq x \leq y$ implies $\|x\| \leq b\|y\|$ with some universal constant $b$ ). Then A has exactly one fixed point $u^{*}$. The iterative sequence $x_{n}=A x_{n-1}$ converges to $u^{*}$ for any initial approximation $x_{0} \in F$. Moreover

$$
\left\|x_{n}-u^{*}\right\|_{X}=o\left(k^{n}\right) \text { as } n \rightarrow \infty
$$

for $\rho(T)<k<1$.

We omit the proof that can be modeled easily on the proof of the Banach Contraction Principle and is essentially founded on the hypothesis of normality for the cone $\mathbb{K}$. In fact this hypothesis is equivalent to the classical policeman lemma ([5]). We can cite many references where it is possible to find the proof of theorems much more general than Theorem 1.3 (to cite only a few [6], [7, [8]).

Remark 1.4. Under the assumptions of Theorem 1.3. the original norm may be replaced by an equivalent norm such that $A$ satisfies the usual Contraction Principle. In fact, given $\varepsilon>0$, by definition of spectral radius we can find $n \in \mathbb{N}$ such that $\left\|T^{n}\right\| \leq(\rho(T)+\varepsilon)^{n}$. Then the norm

$$
\|x\|_{\varepsilon}:=(\rho(T)+\varepsilon)^{n-1}\|x\|_{B}+(\rho(T)+\varepsilon)^{n-2}\|T x\|_{B}+\ldots+\left\|T^{n-1} x\right\|_{B}
$$

is equivalent to the original one in $B$ and

$$
\|T\|_{\varepsilon}-\varepsilon \leq \rho(T) \leq\|T\|_{\varepsilon} .
$$

If $\|x\|_{B}$ is increasing, and this is the case up to passage to equivalent norms since $\mathbb{K}$ is normal $([5])$, then $\|x\|_{\varepsilon}$ is increasing so that Theorem [1.3 still makes sense. Choosing $\varepsilon$ such that $\rho(T)+\varepsilon<1$ we obtain that $A$ is a contraction with respect to the norm $\|] \cdot\left[\|_{\varepsilon}\right.$.

In section 2 we will prove that Theorem 1.1 can be obtained by Theorem 1.3 Moreover this gives a better estimate on the rate of convergence of the sequences approximating the fixed point. Then we give a counterexample to a possible extension of Theorem 1.1.

In section 3, again using Theorem 1.3, we obtain a version of Theorem 1.1 holding for unbounded intervals.

In section 4 the consideration of Volterra type operators gives us the opportunity to prove that the use of a K-norm can also be useful in the case where we know the considered operator to have a contractive iterate. 


\section{A NeW Proof of Theorem 1.1}

Let $T_{0}=0<T_{1}<\ldots<T_{k}=T$ be a partition of the interval $[0, T]=I$. Denote $I_{s}=\left[T_{s-1}, T_{s}\right], \quad s=1, \ldots, k$. We construct a complete K-norm on $C(I, E)$ in the following way:

$$
] u\left[=\left(\sup \left\{\|u(\sigma)\|, \sigma \in I_{1}\right\}, \ldots, \sup \left\{\|u(\sigma)\|, \sigma \in I_{k}\right\}\right) .\right.
$$

The norming space $\mathbb{R}^{k}$ is ordered in the usual way by means of the positive cone. An operator $A$ satisfying the assumptions of Theorem 1.1 is a contraction in the above K-normed space, for a suitable choice of the partition of $[0, T]$, with respect to the positive linear operator $S$ on $\mathbb{R}^{k}$ defined by the following lower triangular matrix:

$$
\left(\begin{array}{ccccc}
\beta+K T_{1}^{1-\alpha} & 0 & 0 & \ldots & 0 \\
K T_{1}^{1-\alpha} & \beta+K \frac{T_{2}-T_{1}}{T_{1}^{\alpha}} & 0 & \ldots & 0 \\
K \frac{T_{1}}{T_{2}^{\alpha}} & K \frac{T_{2}-T_{1}}{T_{2}^{\alpha}} & \beta+K \frac{T_{3}-T_{2}}{T_{2}^{\alpha}} & \ldots & 0 \\
\ldots & \ldots & \ldots & \ldots & \ldots \\
K \frac{T_{1}}{T_{k-1}^{\alpha}} & K \frac{T_{2}-T_{1}}{T_{k-1}^{\alpha}} & \ldots & \ldots & \beta+K \frac{T_{k}-T_{k-1}}{T_{k-1}^{\alpha}}
\end{array}\right) .
$$

In fact we have:

for $s=1$

$$
\begin{gathered}
\sup \left\{\|A u(t)-A v(t)\|, t \in I_{1}\right\} \\
\left.\left.\leq \beta \sup \left\{\|u(t)-v(t)\|, t \in I_{1}\right\}+\sup \left\{\frac{K}{t^{\alpha}} \int_{0}^{t}\|u(\tau)-v(\tau)\| d \tau, t \in\right] 0, T_{1}\right]\right\} \\
\leq\left(\beta+K T_{1}^{1-\alpha}\right) \sup \left\{\|u(t)-v(t)\|, t \in I_{1}\right\},
\end{gathered}
$$

and for $s \in\{2, \ldots, k\}$

$$
\begin{gathered}
\sup \left\{\|A u(t)-A v(t)\|, t \in I_{s}\right\} \\
\leq \beta \sup \left\{\|u(t)-v(t)\|, t \in I_{s}\right\}+\sum_{j=0}^{s-1} \frac{K}{T_{s-1}^{\alpha}} \int_{T_{j}}^{T_{j+1}}\|u(\tau)-v(\tau)\| d \tau \\
\leq \beta \sup \left\{\| u(t)-v\left(t \|, t \in I_{s}\right\}+\sum_{j=0}^{s-1} \frac{T_{j+1}-T_{j}}{T_{s-1}^{\alpha}} \sup \left\{\|u(\tau)-v(\tau)\|, \tau \in I_{j+1}\right\} .\right.
\end{gathered}
$$

In the K-norm symbolism the two previous inequalities amount to saying that for any $u, v \in F$

$$
] A u-A v[\leq S] u-v[.
$$

Now the spectral radius of $S$ is given by

$$
\rho(S)=\max \left\{\beta+K T_{1}^{1-\alpha}, \beta+K \frac{T_{j}-T_{j-1}}{T_{j-1}^{\alpha}}, j=2, \ldots, k\right\} .
$$


Then it is enough to choose the partition of $[0, T]$ in such a way that $\rho(S)<1$ to obtain the thesis of Theorem [1.1 moreover for the iterative sequence $x_{n}$,

$$
\left\|x_{n}-u^{*}\right\|=o\left(k^{n}\right) \quad \text { as } \quad n \rightarrow \infty
$$

for any $k$ such that $\beta<k<1$.

A natural question (which arises from applications, too) is whether Theorem 1.1 continues to hold when the inequality

$$
\|A u(t)-A v(t)\| \leq \beta\|u(t)-v(t)\|+\frac{K}{t^{\alpha}} \int_{0}^{t}\|u(s)-v(s)\| d s, \quad \forall t \in(0, T]
$$

is replaced by the more general one:

$$
\|A u(t)-A v(t)\| \leq \beta\|u-v\|\|u-v\|_{C(I, E)}+\frac{K}{t^{\alpha}} \int_{0}^{t}\|u(s)-v(s)\| d s, \quad \forall t \in(0, T] .
$$

The following counterexample gives a negative answer.

Example 2.1. Consider the operator $A$ of $C(I, R)$ into itself, defined by

$$
A u(t)=\beta \int_{0}^{1} u(s) d s+\gamma \int_{0}^{t} u(s) d s+f(t), \quad \gamma \neq 0
$$

with $f$ a fixed derivable function such that $\int_{0}^{1} f(s) e^{-\gamma s} d s \neq 0$. Easy computations show that $A$ satisfies the above inequality for $0<\beta<1$ and $A$ has a fixed point if and only if $\beta \neq \frac{\gamma}{e^{\gamma}-1}$.

In various papers we have encountered a misuse of this wrong generalization of Theorem 1.1 .

\section{The CASE OF AN UnBOUNDED INTERVAL}

The purpose of this section is to prove that by using a technique similar to that in the previous section we can obtain a result in some sense dual to that of B. Lou for the case of an unbounded interval.

Theorem 3.1. Let $F$ be a closed subset of $B C([1, \infty), E)$ and $A: F \rightarrow F$ an operator. If there exist $\alpha \in(1, \infty), \beta \in[0,1), K \geq 0$ such that for any $u, v \in F$,

$$
\|A u(t)-A v(t)\| \leq \beta\|u(t)-v(t)\|+\frac{K}{t^{\alpha}} \int_{1}^{t}\|u(s)-v(s)\| d s, \quad \forall t \in[1, \infty),
$$

then $A$ has exactly one fixed point $u^{*}$ in $F$. For any $x_{0} \in F$, the iterative sequence $x_{n}=A x_{n-1}$ converges to $u^{*}$ in $F$ and for all $k$ such that $\beta<k<1$,

$$
\left\|x_{n}-u^{*}\right\|=o\left(k^{n}\right) \quad \text { as } \quad n \rightarrow \infty .
$$

The K-normed space suitable for our proof is the space of bounded and continuous functions on $[1, \infty), B C([1, \infty), E)$ equipped with a $\mathrm{K}$-norm taking values in $l^{\infty}$

Consider a partition of the half line $[1, \infty)$ obtained by means of $T_{0}=1<T_{1}<$ $T_{2}<\ldots<T_{k}<\ldots<\infty$ where $T_{k}$ is an increasing sequence and $\lim _{k \rightarrow \infty} T_{k}=\infty$. Let $I_{k}=\left[T_{k}, T_{k+1}\right]$ and define for any $u \in B C([1, \infty), E)$ the following $K-$ norm:

$$
] u\left[=\left(\sup \left\{\|u(s)\|: s \in I_{k}\right\}\right)_{k \in \mathbb{N}} .\right.
$$


Since $\left.\|u\|_{\infty}=\|\right] u\left[\|_{\infty}, B C([1, \infty), E)\right.$ is obviously complete with respect to the above $K-$ norm. Inequalities analogous to the one proved in the previous section gives for any $u, v \in B C([1, \infty), E)$

$$
] A u-A v[\leq S(] u-v[),
$$

where $S$ is the infinite lower triangular matrix whose $k-t h$ row is given by

$$
\left(\frac{T_{2}-T_{1}}{T_{k}^{\alpha}}, \frac{T_{3}-T_{2}}{T_{k}^{\alpha}}, \ldots, \frac{T_{k+1}-T_{k}}{T_{k}^{\alpha}}+\beta, 0, \ldots, 0, \ldots\right):=\left(a_{k, j}\right)_{j \in \mathbb{N}} .
$$

It is simple to see (but it can be found in [3]) that if

$$
\sup _{k} \sum_{j=1}^{\infty}\left|a_{k, j}\right|=\sup _{k}\left(\frac{T_{k+1}-T_{1}}{T_{k}^{\alpha}}+\beta\right)<\infty,
$$

then $S$ is a positive linear operator on $l^{\infty}$ with norm given by (3.1). We are interested in $\|S\|<1$. This can be obtained by the choice of the sequence $\left(T_{k}\right)_{k \in \mathbb{N}}$ we are going to describe.

Let $s$ be a natural integer to be determined in the sequel ( $s$ will depend on $\beta$ ). Define for any $k>1, T_{k}=1+\frac{1}{s}+\frac{1}{s+1}+\ldots+\frac{1}{s+k}$. We have $\frac{T_{k+1}-T_{1}}{T_{k}^{\alpha}} \rightarrow 0$ as $k \rightarrow \infty$, and consequently the sequence $\left(\beta+\frac{T_{k+1}-T_{1}}{T_{k}^{\alpha}}\right)_{k \in \mathbb{N}}$ is bounded and assume its maximum for a suitable $k$ depending on $s$ :

$$
\|S\|=\max _{k}\left(\beta+\frac{T_{k+1}-T_{1}}{T_{k}^{\alpha}}\right)=\beta+\frac{\frac{1}{s}+\frac{1}{s+1}+\ldots+\frac{1}{s+k(s)+1}}{\left(1+\frac{1}{s}+\frac{1}{s+1}+\ldots+\frac{1}{s+k(s)}\right)^{\alpha}} .
$$

For $s \rightarrow \infty$ the above maximum tends to $\beta$ and then we can choose $s$ in such a way that $\beta<\|S\|<1$. Now our statement follows from Theorem 1.2

\section{Volterra operators}

In this section we show that K-norms of the type introduced in sections 2 and 3 can be useful in studying fixed points also in the case of an operator $A$ which is known to have a contractive iterate. We give a very simple example to avoid technicalities which would hide the main idea. Let $A$ be a Volterra integral operator defined by

$$
A u(t)=\frac{1}{t^{\alpha}} \int_{0}^{t} f(s, t, u) d s+g(t), \quad t \in[0, T], 0 \leq \alpha<1,
$$

with $f$ continuous and Lipschitzian with respect to $u$ :

$$
|f(t, s, u)-f(t, s, v)| \leq M|u-v| .
$$

With the same notations used in section 2 we have

$$
] A u-A v[\leq S] u-v[
$$

where the K-norm and the positive matrix $S$ depend on $f$ in a very simple way: really they depend only on the Lipschitz constant $M$. After the construction of the K-norm (i.e. a partition of the interval $[0, T]$ ) and of the matrix $S$, simple iterations of $S$ can give complete information about the number of iterations of $A$ needed to approximate the fixed point with an assigned error estimate. Moreover the error estimate can be assigned to be different from one sub-interval of the partition to the other. 
It turns out that thanks to the fact that the upper extreme in (4.1) is variable, the matrix $S$ is lower triangular, and then dealing with the spectral radius is simple. This would not be the case when working with an integral operator with fixed extremes. This emphasizes again, this time from a $K$ - norm point of view, the well known different behaviors of Volterra and Fredholm integral operators.

\section{REFERENCES}

[1] B. Lou, Fixed points for operators in a space of continuous functions and applications, Proc. Amer. Math. Soc. 127, 2259-2264 (1999). MR 99m:47065

[2] P. P. Zabrejko, K-metric and K-normed linear spaces: a survey, Collect. Math. 48, 825-859 (1997). MR 99a:46010

[3] I. J. Maddox, Elements of Functional Analysis, Cambridge University Press, Cambridge, 1988. MR 90k:46002

[4] I. Rosenholtz, Evidence of a conspiracy among fixed point theorems, Proc. Amer. Math. Soc. 53, 213-218 (1975). MR 53:4036

[5] D. Guo, V. Lakshmikantham, Nonlinear Problems in Abstract Cones, Academic Press, Boston (1988). MR 89k:47084

[6] L. Collatz, Functional Analysis and Numerical Mathematics, Academic Press: Boston, 1966. MR 34:4961

[7] M. Altman, Contractors and equations in pseudometric spaces, Boll. Un. Mat. Ital. 6, 376-384 (1972). MR 47:9376

[8] M. A. Krasnosel'skii, G. M. Vainikko, P. P. Zabreiko, Ya. B. Rutitskij, V. Ya. Stetsenko, Approximate solution of operator equations, Nauka, Fitmatgiz, Moskow (1969); English translation: Wolters-Noordhoff Publishing, Groningen (1972). MR 52:6515

Dipartimento di Matematica, Universitá della Calabria, 87036 Rende (Cosenza), ITALY

E-mail address: e.depascale@unical.it

Dipartimento di Matematica Applicata "U.Dini", Via Bonanno Pisano 25/B, 56126 Pisa, Italy

E-mail address: depascal@dm.unipi.it 